\title{
The Use of a Cementless Tapered Stem in Young Patients Affected of Secondary Hip Arthritis
}

Rafael Llombart Blanco*, Sebastian Cruz and Juan Ramón Valentí

Department of Orthopedic surgery, Clinical Universidad de Navarra, Pio XII 31, 31008 Pamplona, Spain

*Corresponding author: Rafael Llombart Blanco, Department of Orthopedic surgery, Clinical Universidad de Navarra, Pio XII 31 , 31008 Pamplona, Spain, Tel: +34948255400; E-mail: rllombartb@gmail.com

Received: Febuary 24th 2014, Accepted: May 05th 2014, Pub date: May 12th 2014

Copyright: (C) 2014 Blanco RL, et al. This is an open-access article distributed under the terms of the Creative Commons Attribution License, which permits unrestricted use, distribution, and reproduction in any medium, provided the original author and source are credited.

\begin{abstract}
Introduction: The Wagner cone stem was designed for uncemented hip arthroplasty in difficult proximal femoral conditions or femoral deformities. The most common indications for the use of this stem are secondary hip arthritis due to developmental hip dysplasia, septic arthritis or congenital malformations. We present a case series of patients that underwent hip arthroplasty with the use of a Wagner cone stem.

Materials and methods: A retrospective chart review of 17 patients (15 women and 2 men) was performed with a mean age of 39 (25-62). Clinical evaluation was rated with the Merlé D'Aubigné score. Radiographic evaluation consisted in antero posterior and axial views of the hip. One patient was operated bilaterally, with a total of 18 hips operated, and mean follow up of 8 years $(5-12)$.
\end{abstract}

Results: Diagnosis of secondary arthritis was: developmental hip dysplasia in 12 patients, septic arthritis sequelae in 3 patients and other causes of hip arthritis in 3 patients. Average Merlé-D'Aubigné score was 9,9 (8-13) preoperatively and 16,10 (13-18) at last follow-up. One patient presented crural nerve paresia after surgery, which was recovered completely and one stem was reviewed due to aseptic loosening.

Discussion: The Wagner cone stem has successfully resolved cases with abnormalities in the proximal femur in patients with secondary hip arthritis. Although this procedure is not exempt of complications, it has shown good stability which translates into good results in the medium to long term.

Keywords: Hip Arthritis; Hip Arthoscopy

\section{Introduction}

Hip osteoarthritis as the consequence of a previous pathology of the hip has been classically defined as secondary osteoarthritis. The main cause of secondary arthritis in the hip is developmental hip dysplasia (DDH). DDH includes a spectrum of disorders ranging from mild dysplasia of the acetabulum or femur to high dislocation of the hip [1] Patients with DDH present with abnormal anatomy affecting the acetabulum, the femur and the surrounding soft tissues. The femur is hypoplastic with a narrow medullary canal and torsion may be affected in terms of anteversion and a posterior greater trochanter [2]. These anatomical variations and the fact that many patients might have undergone previous surgery make total hip arthroplasty in patients with DDH a challenge procedure. But there are other causes of secondary hip arthritis that could modify the anatomy of the femur like septic arthritis sequelae, tuberculosis of the hip, congenital coxa vara, fractures, bone dystrophies, previous surgeries of the proximal femur and congenital malformations [3].

Patients with secondary arthritis present symptoms at younger age than those affected with primary osteoarthritis. That is another concern for the orthopedic surgeon. Firstly joint-preserving procedures should be considered in young adults, but when advanced osteo-arthritic changes are present total joint arthroplasty is the final option [1]. When planning a total hip arthroplasty in an arthritic hip secondary to a dysplasia, these abnormalities and the age of patient must be taken into account. In terms of choice of implant, there is a variety of options. The use of modular versus straight stems and cemented or uncemented stems are still on debate.

In 1990 Wagner designed the Wagner Cone Prosthesis Hip Stem (Zimmer ${ }^{\bullet}$ ), which is a modification of the Wagner revision stem. It was designed for uncemented fixation in complicated bone conditions of the proximal femur or femoral deformities [4]. The stem has a conical shape and cone progression angle of $5^{\circ}$. The lack of a proximal flare enables placement within the narrow medullary cavity.

A circular cross-sectional area enables intra-operative correction of excessive version in the femur. 8 longitudinal ribs provide optimal rotational stability for primary fixation. Theses ribs also favor osteointegration. Due to its circular cross-section, the cone prosthesis can be introduced into the femur at any required angle of anteversion, and intraoperative corrections are easily executed, if necessary [5].

The purpose of this study is to show the functional results of patients affected of secondary hip arthritis treated with total hip arthroplasty using an uncemented tapered stem (Wagner Cone Prosthesis Hip Stem Zimmer ${ }^{\circledR}$. 
Citation: Blanco RL, Cruz S, Valentí JR (2014) The Use of a Cementless Tapered Stem in Young Patients Affected of Secondary Hip Arthritis. J

\section{Material and Methods}

Patients that underwent total hip arthroplasty with diagnosis of secondary hip arthritis have been reviewed.

\section{Patients}

Between 1997 and 2009, 17 patients (15 women and 2 men) aged 25 to 62 (mean 39) underwent a total hip arthroplasty with a Wagner cone stem. One patient had a bilateral hip arthroplasty (Figure 1). Of the 17 patients, 12 had a development dysplasia of the hip, was performed. One patient had a bilateral hip arthroplasty. 3 had sequelae of septic arthritis, 2 had post-traumatic arthritis and 1 had spondylo epiphyseal dysplasia. 5 patients had undergone 3 or more surgeries, one patient 2 previous surgeries, 3 patients one surgery and 8 patients had never been operated before. All these data have been summarized in Table 1.

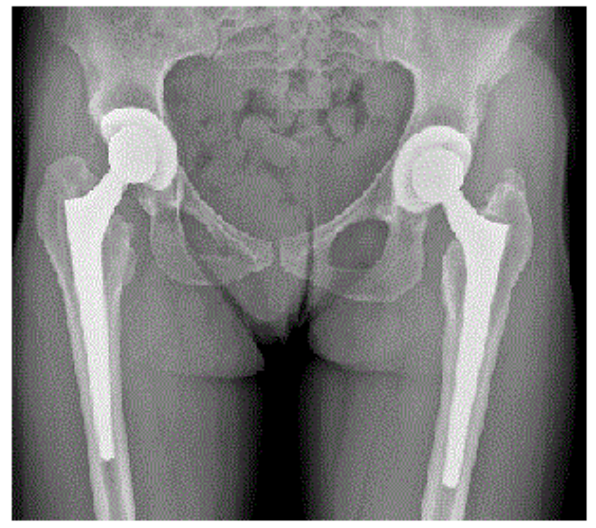

Figure 1: A 39 year female patient with diagnosis of DDH who had bilateral total hip arthroplasty.

\begin{tabular}{|c|c|c|c|c|c|}
\hline Patient & Sex & Age & Diagnosis & Crowe classification & Clinical dismetry $(\mathrm{cm})$ \\
\hline 1 & $\mathrm{~F}$ & 31 & $\mathrm{DDH}$ & 1 & 0 \\
\hline 2 & $\mathrm{~F}$ & 42 & Septic arthritis & III & 4.5 \\
\hline 3 & M & 33 & Septic arthritis & II & 2 \\
\hline 4 & $\mathrm{~F}$ & 26 & DDH & III & 3 \\
\hline 5 & $\mathrm{~F}$ & 51 & SED & II & 2 \\
\hline 6 & $\mathrm{~F}$ & 39 & DDH & 1 & 0 \\
\hline 7 & $\mathrm{~F}$ & 33 & DDH & II & 2 \\
\hline 8 & $\mathrm{~F}$ & 25 & Septic arthritis & 1 & 0 \\
\hline 9 & $\mathrm{~F}$ & 35 & $\mathrm{DDH}$ & II & 2 \\
\hline 10 & $\mathrm{~F}$ & 35 & $\mathrm{DDH}$ & 1 & 0 \\
\hline 11 & $\mathrm{~F}$ & 45 & DDH bilateral & III & 1.5 \\
\hline 12 & $\mathrm{~F}$ & 48 & DDH & II & 0.5 \\
\hline 13 & $\mathrm{~F}$ & 56 & DDH & IV & 7.5 \\
\hline 14 & $\mathrm{~F}$ & 42 & DDH & 1 & 0.5 \\
\hline 15 & $\mathrm{~F}$ & 44 & Acetabular fracture & 1 & 0 \\
\hline 16 & M & 62 & Post traumatic arthritis & 1 & 0 \\
\hline 17 & $\mathrm{~F}$ & 30 & $\mathrm{DDH}$ & 1 & 0 \\
\hline
\end{tabular}

Table 1: Summary of preoperative patient's data

\section{Surgical technique:}

All patients received the same antibiotic prophylaxis (cephazolin $2 \mathrm{~g}$ 30 minutes prior to the surgery and $1 \mathrm{~g}$ every eight hours during the 24 hours post-surgery) and low molecular weight heparin perioperatively as antithrombotic prophylaxis. A modified Watson-Jones anterolateral approach was performed in all cases. Osteotomy of the trochanter was not performed. After capsulotomy the version of the femoral neck was assessed intraoperative. The acetabulum was prepared and the appropriate acetabular component was implanted. 9 patients had an uncemented press-fit cup, 2 had expansive cups and in one case a cemented cup was implanted. Two cases needed of autograft 
Page 3 of 4

augmentation in the acetabulum. In all patients a Wagner cone stem was implanted.

To evaluate the clinical status of patients we used the Merlé D 'Aubigné score [6]. For the radiographic analysis an anteroposterior and axial view of the hips were used at each postoperative follow up. Radiographic signs of loosening were identified and recorded.

\section{Results}

The mean follow-up duration was 8 years (5-12). The Merle D 'Aubigné score improved from 9.9 (8-13) preoperatively to 16.10 (13-18) in the last follow-up. One patient presented crural nerve paresia immediately after surgery that was completely recovered after three months. There were no dislocations after surgery, No other complications such as deep venous thrombosis, wound infection or peri prosthetic fractures were registered. The radiological evaluation did not show any heterotopic ossifications in any of the patients. Osteo integration was seen in almost every patient except from one who presented a stem subsidence 15 months after surgery (Figure 2) with radiolucent lines $>5 \mathrm{~mm}$. Clinical and imaging tests revealed an as septic loosening of the stem. The patient underwent a revision surgery and the Wagner cone stem was replaced with a CLS Spotorno press fit stem.

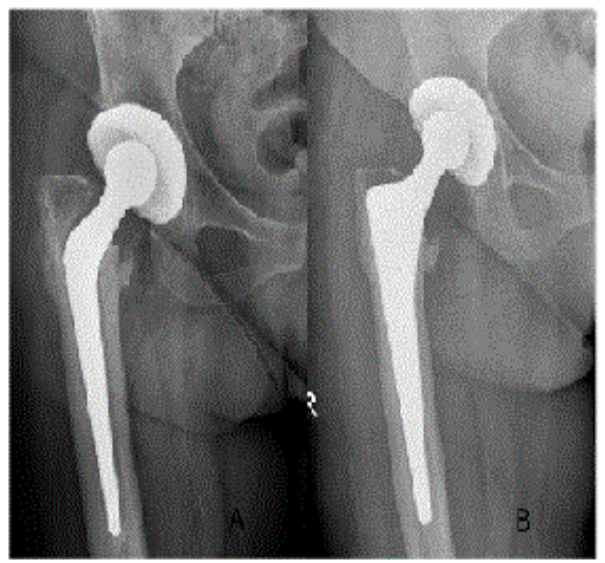

Figure 2: A 62 year female who had a stem subsidence 15 months after her total hip arthroplasty.

\section{Discussion}

In our study we found an improvement in the functional status after a total hip arthroplasty with the use of a Cementless tapered stem (Wagner cone stem) with a $95 \%$ of survival of the stems after a mean follow up of 8 years. Our results are consistent with other previous published studies both in short and long terms [7-13].

Some authors have pointed out intraoperative fractures, dislocations and infection as their main complications using the cone stem [14]. In our series we found one minor complication which was a temporary crural nerve paresia. Nerve palsy was initially to be caused by leg lengthening in patients with $\mathrm{DDH}$. Eggli [15] observed that there was a significant correlation between difficulty and nerve damage, but the correlation between leg lengthening and nerve lesions was not significant.
The major complication in our series was one case with aseptic loosening of the stem 15 months after surgery. Early subsidence has previously been reported, in our series the only case that presented subsidence ended in revision surgery. Ström showed that a few stems subsided to a very small degree in the first months, but remained stable thereafter [6]. Most of the published works show excellent osteointegration and stability of the tapered stem. It is believed that the tapered geometry permits a load distribution per surface that is greater in the proximal portion of the femur that avoids stress-shielding [13]. Stability of the stems could lead to the discussion of whether to use cemented stems. Ström [16] compared the use of uncemented cone prosthesis with the cemented Biemtric stem in young patients with osteoarthritis. They concluded that both were stable and had excellent clinical results after two years. A recent published meta-analysis showed that there are no differences in terms of implant survival as measured by the revision rate but better short-term clinical outcome, particularly an improved pain score can be obtained with cemented fixation [14].

Version of the altered femur is other issue that has been well managed with the use of the Wagner cone stem. The position is not determined by the anatomical characteristics of the dysplastic femoral epiphysis [16], the circular section of the tapered stem, provided with 8 longitudinal sharp ribs, allows the surgeon to choose the degree of anteversion that he considers as better. Modular stems have been proposed as a solution to version, length and offset problems. A retrospective study of 61 modular neck prostheses found a survival rate of $97.5 \%$ at 11 years [17]. Perka [8] found a survival rate at 9,3 years with the use of an straight tapered stem. The cone prosthesis is a non-modular structure and therefore less expensive [9].

The weakness of this study is due to its several limitations. Most important one is that it is a retrospective study of a case series of patients mostly affected of developmental dysplasia of the hip with a medium follow up.

After this study we conclude that the uncemented tapered Wagner stem has successfully resolved cases with abnormalities in the proximal femur in young patients with secondary hip arthritis. Although this procedure is not exempt of complications, it has shown good stability which translates into good results in the medium to long term.

\section{References}

1. Kosuge D, Yamada N, Azegami S, Achan P, Ramachandran M (2013) Management of developmental dysplasia of the hip in young adults: current concepts. Bone Joint J 95-95B: 732-7.

2. Sugano N1, Noble PC, Kamaric E, Salama JK, Ochi T, et al. (1998) The morphology of the femur in developmental dysplasia of the hip. J Bone Joint Surg Br 80: 711-719.

3. Boisgard S, Descamps S, Bouillet B (2013) Complex primary total hip arthroplasty. Orthop Traumatol Surg Res 99: S34-42.

4. Wagner H, Wagner M (2000) Cone prosthesis for the hip joint. Arch Orthop Trauma Surg 120: 88-95.

5. Kim YY, Kim BJ, Ko HS, Sung YB, Kim SK, et al. (1998) Total hip reconstruction in the anatomically distorted hip. Cemented versus hybrid total hip arthroplasty. Arch Orthop Trauma Surg 117: 8-14.

6. D'AUBIGNE RM, POSTEL M (1954) Functional results of hip arthroplasty with acrylic prosthesis. J Bone Joint Surg Am 36-36A: 451-75.

7. Ström H, Mallmin H, Milbrink J, Petrén-Mallmin M, Nivbrant B, et al. (2003) The cone hip stem: a prospective study of 13 patients followed for 5 years with RSA. Acta Orthop Scand 74: 525-530. 
Citation: Blanco RL, Cruz S, Valentí JR (2014) The Use of a Cementless Tapered Stem in Young Patients Affected of Secondary Hip Arthritis. J Arthritis 3: 1000128. doi:10.4172/2167-7921.1000128

Page 4 of 4

8. Perka C, Fischer U, Taylor WR, Matziolis G (2004) Developmental hip dysplasia treated with total hip arthroplasty with a straight stem and a threaded cup. J Bone Joint Surg Am 86-86A: 312-9.

9. Pak P, de Steiger R (2008) Cone femoral prosthesis for osteoarthritis of the hip with femoral dysplasia. J Orthop Surg (Hong Kong) 16: 206-210.

10. Bruzzone M1, La Russa M, Garzaro G, Ferro A, Rossi P, et al. (2009) Long-term results of cementless anatomic total hip replacement in dysplastic hips. Chir Organi Mov 93: 131-136.

11. Schuh A, Schraml A, Hohenberger G (2009) Long-term results of the Wagner conical screw cup. Int Orthop 33: 659-664.

12. Claramunt RT, Marqués F, León A, Vilà G, Mestre C, et al. (2011) Total hip replacement with an uncemented Wagner cone stem for patients with congenital hip dysplasia. Int Orthop 35: 1767-1770.

13. Faldini C, Miscione MT, Chehrassan M, Acri F, Pungetti C (2011) Congenital hip dysplasia treated by total hip arthroplasty using Cementless tapered stem in patients younger than 50 years old: results after 12-years follow-up. J Orthop Traumatol 12 :213-218.
14. Abdulkarim A, Ellanti P, Motterlini N, Fahey T, O'Byrne JM (2013) Cemented versus uncemented fixation in total hip replacement: a systematic review and meta-analysis of randomized controlled trials. Orthop Rev (Pavia) 5: e8.

15. Eggli S, Hankemayer S, Müller ME (1999) Nerve palsy after leg lengthening in total replacement arthroplasty for developmental dysplasia of the hip. J Bone Joint Surg Br 81: 843-845.

16. Ström H, Kolstad K, Mallmin H, Sahlstedt B, Milbrink J (2006) Comparison of the uncemented Cone and the cemented Bimetric hip prosthesis in young patients with osteoarthritis: an RSA, clinical and radiographic study. Acta Orthop 77: 71-78.

17. Traina F1, De Clerico M, Biondi F, Pilla F, Tassinari E, et al. (2009) Sex differences in hip morphology: is stem modularity effective for total hip replacement? J Bone Joint Surg Am 91 Suppl 6: 121-128. 